\title{
A virtual tour of the Galilean Satellites
}

\author{
Paul Schenk \\ Lunar and Planetary Institute, Houston \\ USA \\ email: schenk@lpi.usra.edu
}

\section{Introduction: the four galilean satellites}

Galileo's imagination was quick to comprehend the importance of the 4 starry objects he observed near Jupiter in January 1610, not only for himself as a scientist but for our common understanding of the place of the Earth and our species in the cosmos. Even he, however, could not have imagined what those four objects would actually look like once humans got their first good look. Some 369 years the fast traveling Voyager 1 and 2 spacecraft provided that first good look during 1979, followed by an even closer look from the Galileo Orbiter beginning in 1996 through 2001. The following mosaics represent some of the best of those views. They include views of impact craters young and ancient, icy terrains that have been intensely faulted, eroded or disrupted, mountains towering 10 or more kilometers high, and volcanic eruptions hotter than those on Earth. Each of the four Galilean satellites is geologically distinct, betraying very diverse global histories and evolutions. Images and other observations of these 4 objects revealed the importance of tidal heating and subsurface water oceans in planetary evolution, but mapping is very incomplete. New missions to explore these planetary bodies are being planned and the images and observations of the missions that went before will lay the groundwork for these new explorations as we begin the $5^{\text {th }}$ Galilean century.

\section{The four galilean satellites}

This montage (Figure 1) shows each of the four Galilean satellites at different scales. From left to right the satellites are: Io, Europa, Ganymede, and Callisto. The top two rows show global views, one with cut-away views showing our best concepts of what the interiors likely look like. Although each has a rocky deep core, ice-rich crusts and mantles of differing depths dominate the outer three satellites. The bottom views show how the surface changes as we zoom in on selected features at progressively higher resolution. Each row down represents an increase in resolution of roughly a factor of ten: the first two rows are at 10 kilometers, increasing to $\sim 1$ kilometer, $\sim 100$ meters, and finally $\sim 10$ meters (bottom). What stands out is the diversity of features at different scales and on the different satellites.

\section{Callisto}

CALLISTO GLOBAL (LEADING HEMISPHERE)

Most of the large multiring impact basins that characterize Callisto formed on this hemisphere (Figure 2). Linear crater chains were formed by disrupted comets, which strike Callisto while outbound after a close passage to Jupiter. Also visible are innumerable bright spots, the marks of countless impact craters on this ancient surface. Orthographic Projection: Center $0^{\circ} \mathrm{N}, 90^{\circ} \mathrm{W}$. 


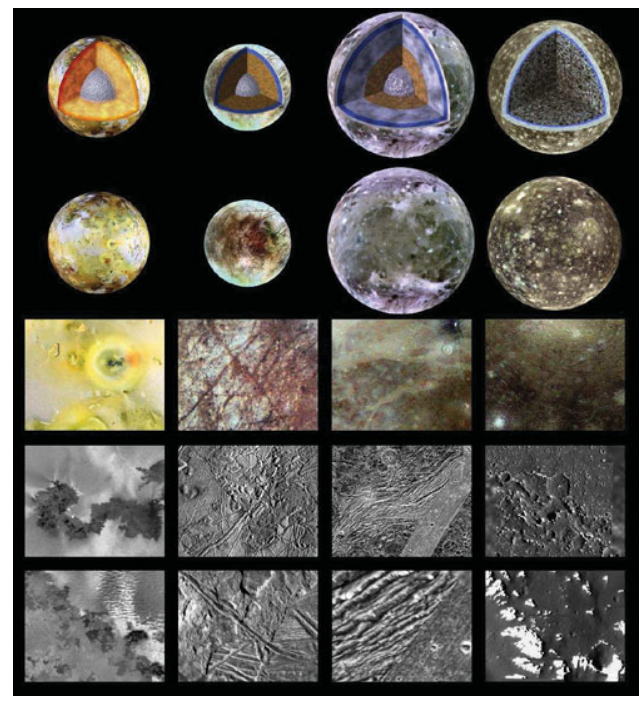

Figure 1.

\section{CALLISTO: ASGARD MULTIRING BASIN}

This spectacular radial swath (Figure 3) shows the transition from Asgard center (including floor and rim deposits) to ancient cratered plains. The prominent 115-kilometer-wide penedome impact crater Doh obscures the center of Asgard at the top of the mosaic. A large intensely fractured central dome 25-kilometers-wide dominates Doh. 1500-metershigh arcuate ridges in the upper half of the mosaic are inner rings of the Asgard structure. Darker areas to the south are heavily cratered plains, crossed by two of the basin's outer graben rings. Small landslides can be also seen within some of these craters. This terrain is generally degraded and partly eroded. The mosaic is $\sim 800$ kilometers long. ENCOUNTER: Galileo C10 RESOLUTION: 90 meters/pixel.

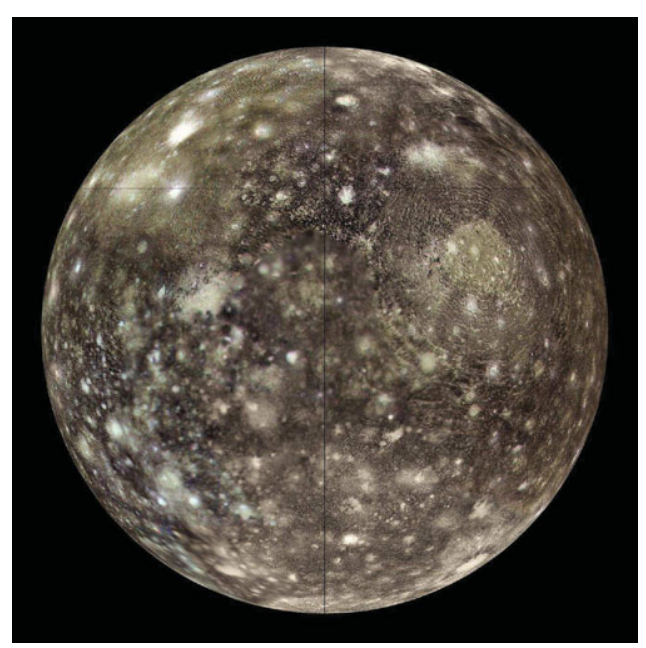

Figure 2. 


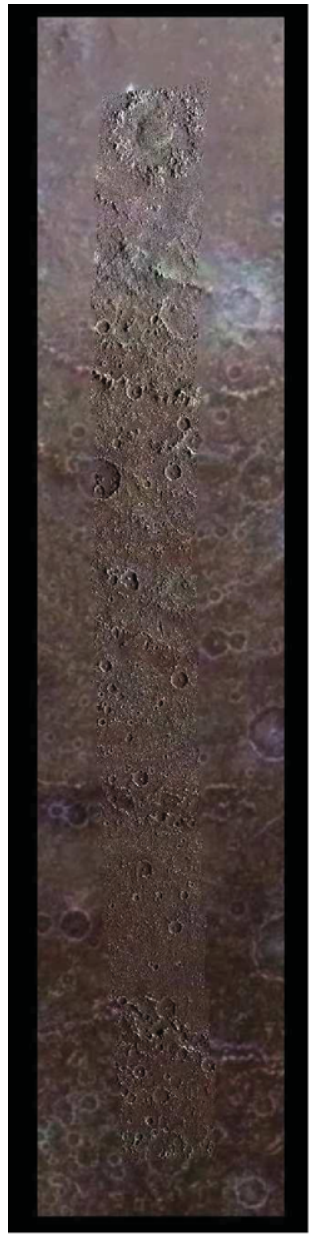

Figure 3 .

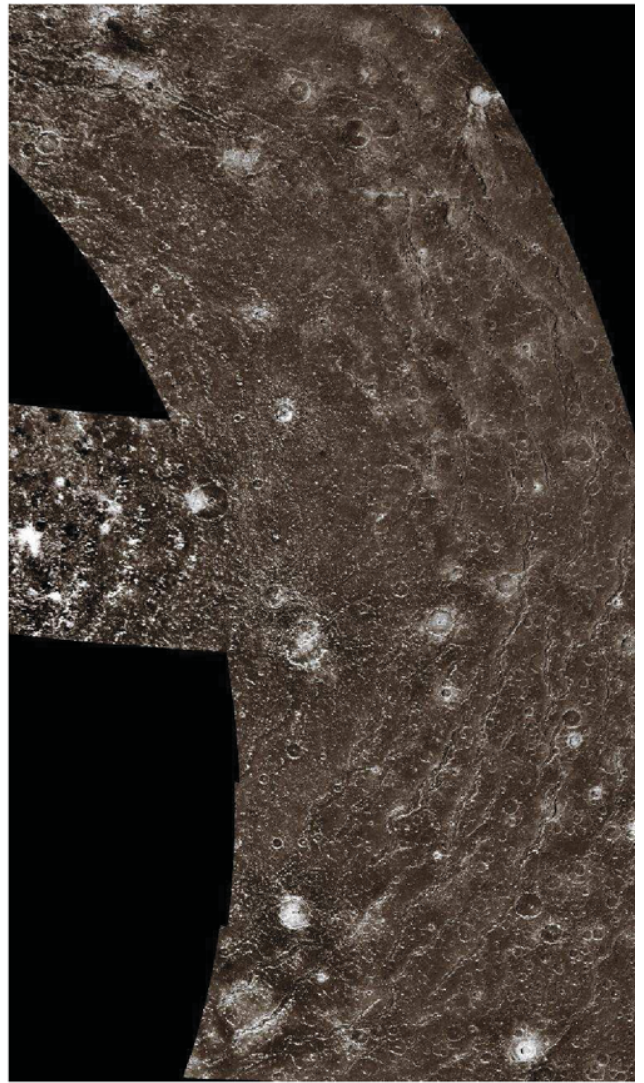

Figure 4 .

\section{CALLISTO: VALHALLA MULTIRING BASIN}

Galileo acquired this multiframe mosaic (Figure 4) of the eastern half of Valhalla to further investigate how this global-scale event formed. The central zone at left is a relatively bright but rugged circular patch, probably formed by refrozen impact deposits on the floor of the impact. Unlike lunar basins there is no deep central depression here. Outward up to 2000 kilometers from the center extends a zone numerous concentric ridges, many of which retain relief of 2 to 3 kilometers, transitioning to graben and scarps superposed on cratered plains. Although the original crater rim can no longer be identified, the size of the original impact crater is estimated to be approximately 1000 kilometers. ENCOUNTER: Galileo C9 RESOLUTION: 410 meters/pixel

\section{CALLISTO: PERSPECTIVE VIEWS}

These four views (Figure 5) show the relief of Callisto from different perspectives. These renderings are made digitally by combining the original image mosaics with topographic maps of the surface. Vertical exaggeration in these views is 10 to 30 times normal. 


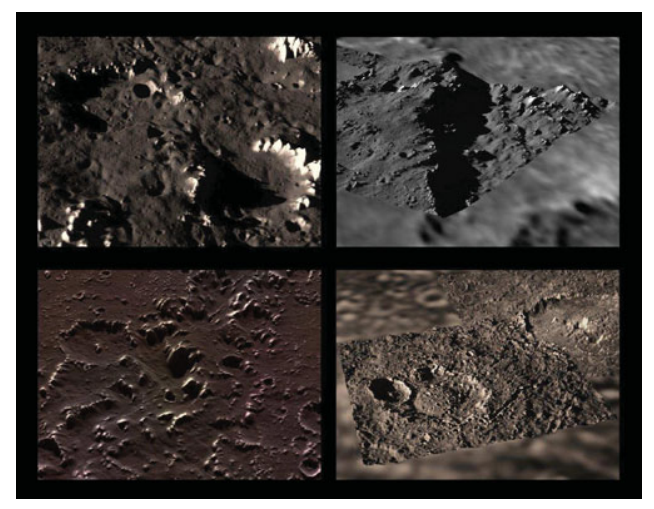

Figure 5.

Upper left: Cratered Plains - Very High Resolution.

This is one of the highest resolution views we have of Callisto. Most of the icy surface is gone, replaced by a dark non-icy layer that is now heavily cratered and has a rugged topography. The larger older craters are partly eroded, leaving sharp-peaked knobs several hundred meters high.

ENCOUNTER: Galileo C21 RESOLUTION: 15 meters/pixel.

Upper right: Valhalla - Outer Ring Scarp.

Our best observation of one of the Valhalla basins many rings shows a prominent fault scarp over 1700 meters high. Several minor fault scarps and aligned knobs are also visible on the floor of the graben itself (although we do not see the opposing wall scarp), indicating that these terrains also experienced faulting.

ENCOUNTER: Galileo C3 RESOLUTION: 46 meters/pixel

Lower right: Hár and Tindr.

This combined pair of images compares craters of different ages. Tindr, the younger large central pit crater to the right is 70 kilometers wide and $\sim 1$ kilometer deep. Radial textures outside the rim are due to ejecta blasted out during impact that scoured nearby surfaces. The ancient impact scar Hár is the concentric set of degraded topographic features. Surrounding the 25-kilometer-wide smooth rounded central dome is a 15-kilometer-wide ring depression and an annular plateau. The dome and ring depression are part of the central uplift complex and the annular plateau is the remains of the crater floor impact deposit. Tindr:

ENCOUNTER: Galileo C10 RESOLUTION: 400 meters/pixel Hár:

ENCOUNTER: Galileo C9 RESOLUTION: 145 meters/pixel

Lower left: Asgard graben.

This view of the outermost ring graben at the Asgard impact basin shows the erosion that has partly destroyed older topography, including the graben walls that curve across the center of the view, as well as older crater rims. Total relief across the graben is roughly 1.5 kilometers.

ENCOUNTER: Galileo C10 RESOLUTION: 90 meters/pixel 


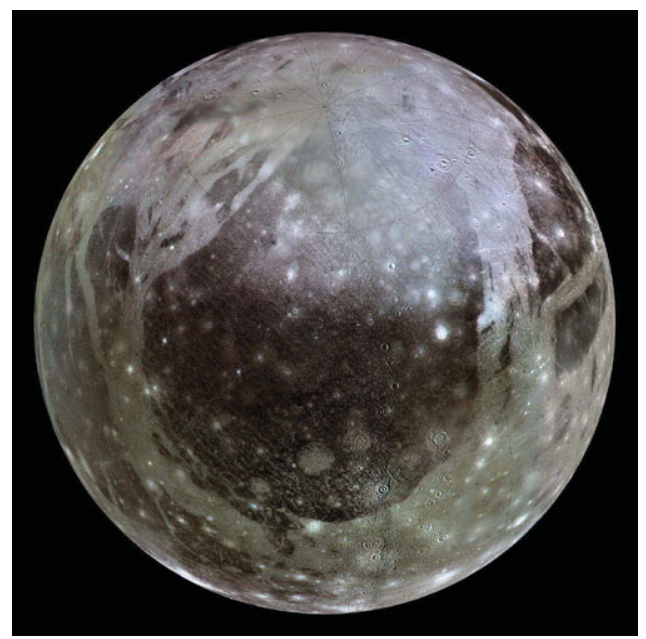

Figure 6 .

\section{Ganymede}

\section{GANYMEDE GLOBAL (GALILEO REGIO, POLAR CAP)}

The huge dark feature in this view (Figure 6), Galileo Regio, is the largest contiguous geologic feature on Ganymede. Astronomers may have seen Galileo Regio and Pioneer saw but did not resolve it. Galileo Regio is a large block of older dark terrain, predating the bands of icy volcanic and tectonic bright terrain surrounding it. Remnants of dark terrain now cover only $33 \%$ of Ganymede. Arcuate furrows formed during a very large ancient impact event. The bright polar frost cap is believed due to sputtering and redistribution of water ice controlled by Ganymede's magnetic field.

Orthographic Projection: Center $42^{\circ} \mathrm{N}, 130^{\circ} \mathrm{W}$.

GANYMEDE: NIPPUR SULCUS This three-frame Galileo mosaic (Figure 7) shows several crosscutting bands of bright terrain that have been deformed to different degrees. The smooth narrow diagonal band Nippur Sulcus was the last formed and least deformed. Smoothness is consistent with volcanic resurfacing by water lavas. To the north lies a wide zone of intensely grooved and fractured bright terrain. Dark terrain to the south is itself heavily deformed by numerous narrow grooves and fissures. Impact craters have been relaxed or distorted, indicative of higher heat flow in the distant past.

ENCOUNTER: Galileo G2 RESOLUTION: 100 meters/pixel

\section{GANYMEDE: NICHOLSON REGIO AND ARBELA SULCUS}

This 9-frame mosaic (Figure 8) illustrates the complex geologic history of these ancient terrains. Belts of narrow fractures can be seen, especially towards the south and east. Most (though not all) large craters are degraded, either by fracturing and disruption, landform degradation, viscous relaxation, or a combination of these. Relatively bright circular patches are probably ancient impact scars. Two bands of bright terrain cross Nicholson Regio here, including the smooth bright band Arbela Sulcus across scene center. Most lanes of bright terrain formed by flooding by icy lavas and tectonic fracturing. The scene is $\sim 600$ kilometers across.

ENCOUNTER: Galileo G7 RESOLUTION: 180 meters/pixel

ENCOUNTER: Galileo G28 RESOLUTION: 132 meters/pixel 


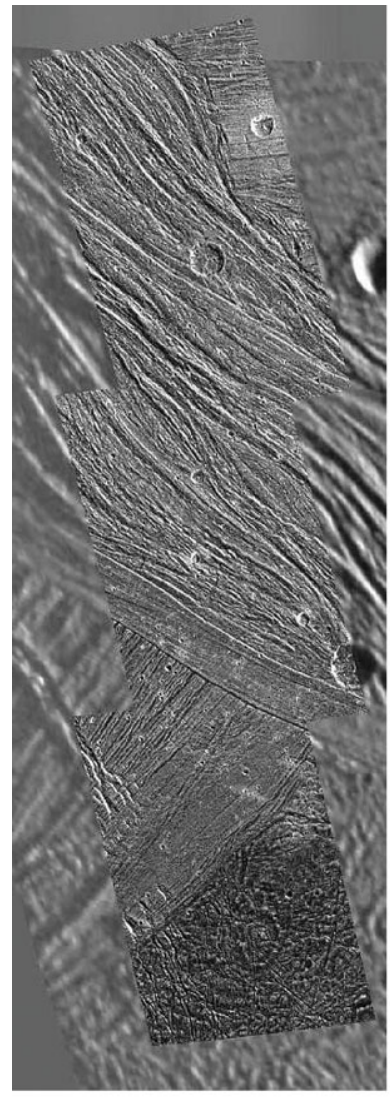

Figure 7.

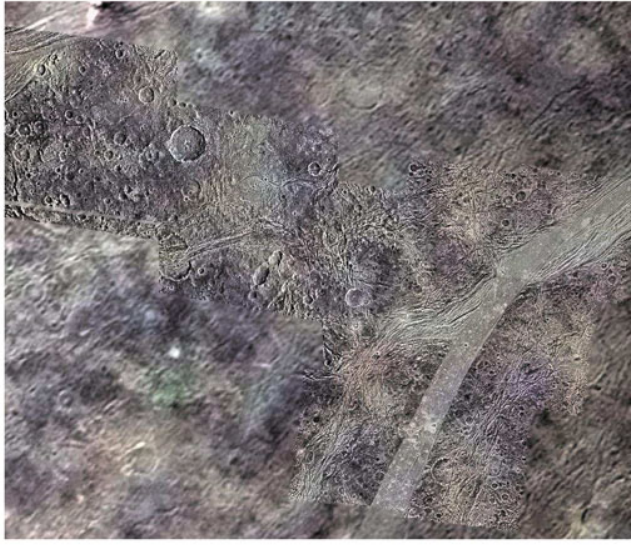

Figure 8 .

GANYMEDE: CALDERAS This six-frame mosaic of bright terrain features 6 irregularly shaped rimmed depressions interpreted to be volcanic calderas (Figure 9). Most are cut by the narrow band of smooth bright terrain crossing the scene east to west. The largest caldera has a rugged surface characteristic of thick viscous lavas. The smooth

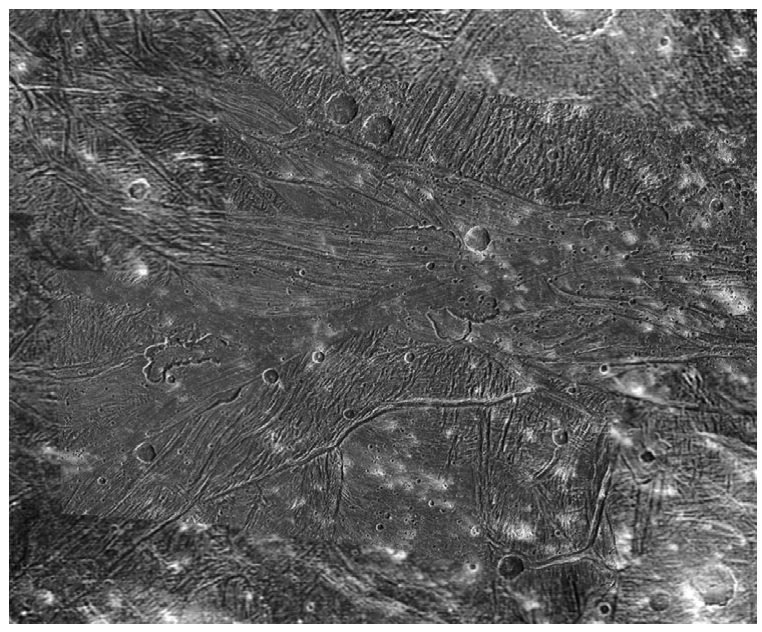

Figure 9. 


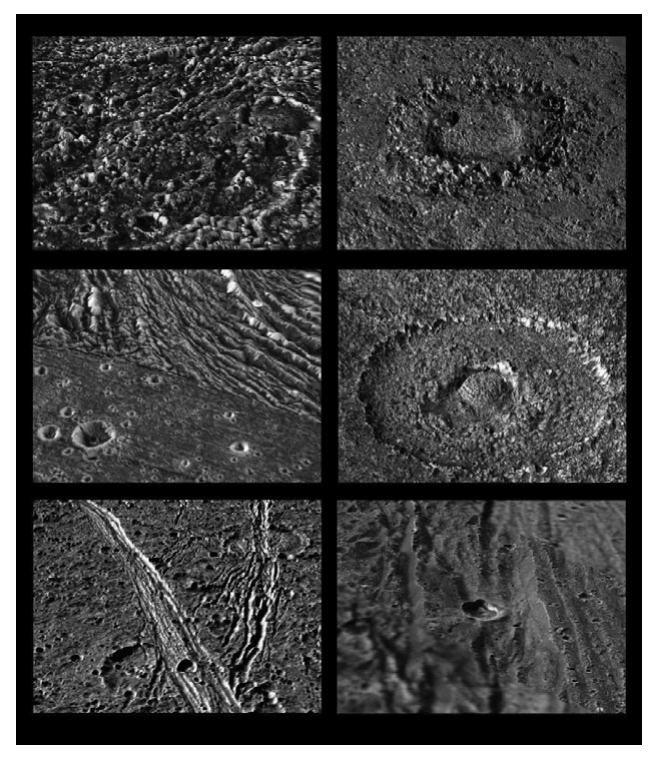

Figure 10.

surface of the band, in contrast, is indicative of runny watery lavas. At least 40 calderas have been identified globally, indicating that volcanism played an important role in bright terrain. Secondary craters from Osiris form the bright splotches. The scene is $\sim 600$ kilometers across.

ENCOUNTER: Galileo G8 RESOLUTION: 180 meters/pixel

\section{GANYMEDE: PERSPECTIVE VIEWS}

These six views show the relief of Ganymede from different perspectives (Figure 10). These renderings are made digitally by combining the original image mosaics with topographic maps of the surface. Vertical exaggeration in these views is 10 to 30 times normal.

\section{Upper left: Dark terrain - Galileo Regio.}

This observation of dark terrain is centered near the intersection of several furrows. The 1-to-2 kilometer deep furrows (or graben) are part of an ancient giant impact. Most features were originally deeper and have been modified by viscous creep of ice at higher temperatures than are prevalent now. Bright and dark materials are also partly segregated by downslope creep into bright slopes and peaks and darker valleys and low areas. ENCOUNTER: Galileo G1 RESOLUTION: 77 meters/pixel ENCOUNTER: Galileo G2 RESOLUTION: 90 meters/pixel

Upper right: Penedome crater Neith.

A 1-kilometer-high, 45-kilometer-wide central dome dominates Neith and surrounding ring of massifs but the crater rim normally present is mostly absent. Penedome craters like Neith no longer form today. The unusual morphology is related to higher heat flow in the ancient past of Ganymede and Callisto.

ENCOUNTER: Galileo G7 RESOLUTION: 140 meters/pixel

Center left: Bright terrain - Arbela Sulcus.

This view shows the truncation of a band of intensely faulted bright terrain (at top) by the smooth band of Arbela Sulcus in the foreground. Ridges are typically 200 meters 
high, the tallest almost 500 meters. The largest crater is $\sim 500$ meters deep and 2.5 kilometers across.

ENCOUNTER: Galileo G7

RESOLUTION: 180 meters/pixel

ENCOUNTER: Galileo G28

RESOLUTION: 132 meters/pixel

Center right: Central dome crater - Melkart.

Melkart, a 100-kilometer-wide central dome crater, is relatively young and well preserved. The central dome is $\sim 30$ kilometers wide and $\sim 1$ kilometer high. Domes like this, seen only on Ganymede and Callisto, may be material uplifted from several kilometers deep during impact, exposing Ganymede's interior.

ENCOUNTER: Galileo G8 RESOLUTION: 180 meters/pixel

Lower left: Dark Terrain and Anshar Sulcus- Marius Regio.

Several generations of fracture sets cut across Marius Regio in this area, with the most prominent of these leading to the formation of the narrow lane of grooved or fractured bright terrain. Dark terrain features several craters 20 to 30 kilometers across that have been fractured and topographically relaxed.

ENCOUNTER: Galileo G8 RESOLUTION: 145 meters/pixel

Lower right: Bright and dark Terrain - Harpagia Sulcus.

This view shows the boundary between dark terrain (left) and bright terrain (right). Here the boundary has been faulted in several places as the icy crust has been stretched, forming relatively steep cliff walls several hundred meters high within both dark terrain and younger bright terrain.

ENCOUNTER: Galileo G28 RESOLUTION: 20 meters/pixel

\section{Europa}

EUROPA GLOBAL (ICE RIFTING) This global view of Europa (Figure 11) highlights a vast zone of dark dilational bands known as Argadnel Regio forming the largest, most coherent zone of rifting evident on Europa today. These short stubby dark bands

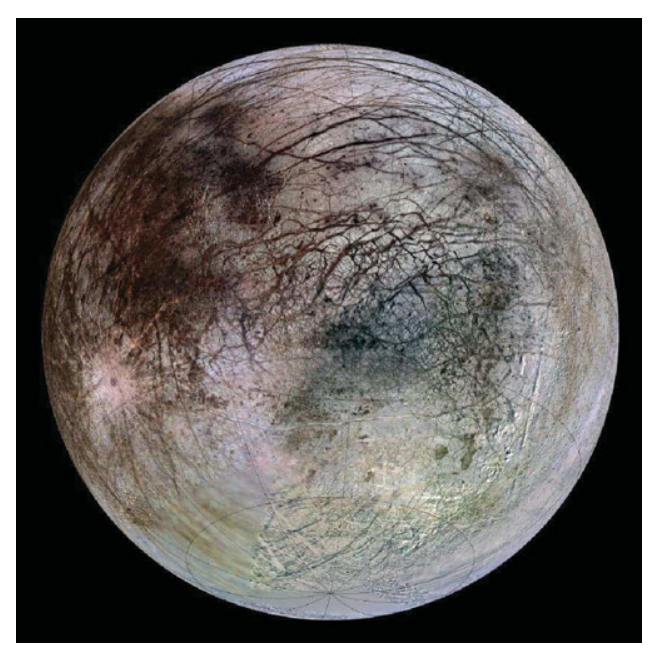

Figure 11. 
(formerly known as wedge-shaped bands) are typically 25 kilometers across and up to 300 kilometers long and formed when the icy shell cracked and was pulled apart in a north-south direction. The bright band Agenor Linea at south center runs along the southern border of this rift zone. Long dark arcuate bands (formerly known as triple bands) dominate the regions north of the dilational bands. Many of these features may have formed together, possibly during an episode of polar wander on Europa.

Orthographic Projection: Center $25^{\circ} \mathrm{S}, 215^{\circ} \mathrm{W}$.

\section{EUROPA - POLE-TO-POLE}

This pole-to-pole mosaic (Figure 12), was obtained during four orbits: E11 to the west, E15 to the north, E19 to the far north, and E17 to the south. Here we see a transition from ridged plains up north featuring long narrow bands and small dark spots (probably the surface expressions of diapirs) to disrupted terrains near the equator, as well as a number of narrow dark dilational bands. Ridged plains lie to the south, as do the large dark spots Thrace and Thera Macula.

ENCOUNTER: Galileo E11 RESOLUTION: 220 meters/pixel ENCOUNTER: Galileo E15 RESOLUTION: 230 meters/pixel ENCOUNTER: Galileo E17 RESOLUTION: 225 meters/pixel ENCOUNTER: Galileo E19 RESOLUTION: 200 meters/pixel

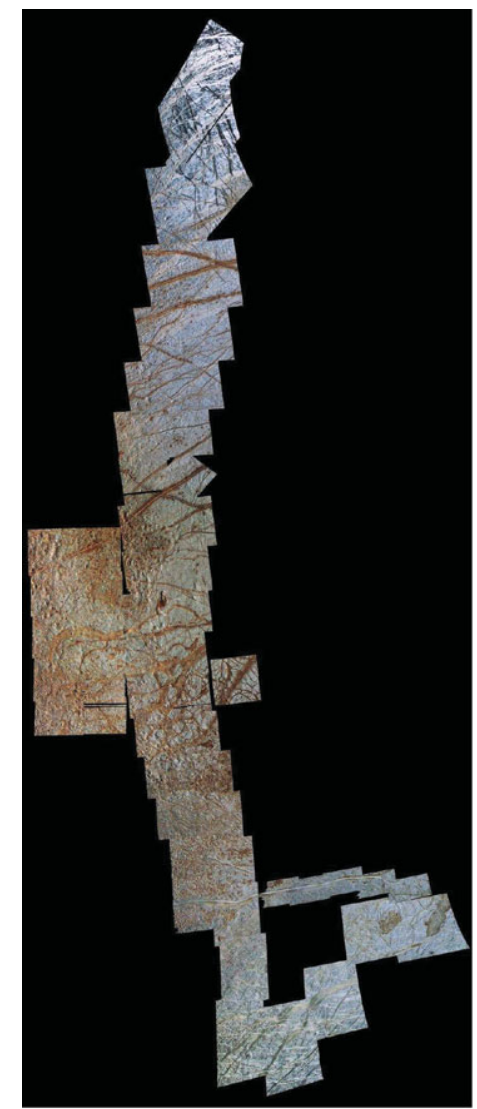

Figure 12.

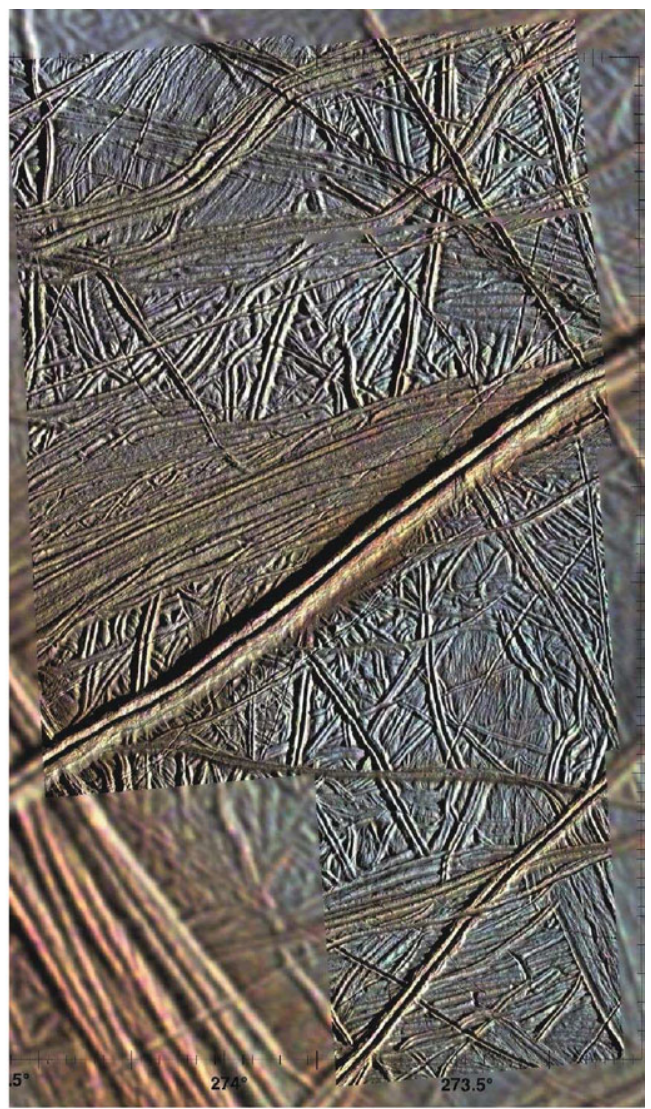

Figure 14. 


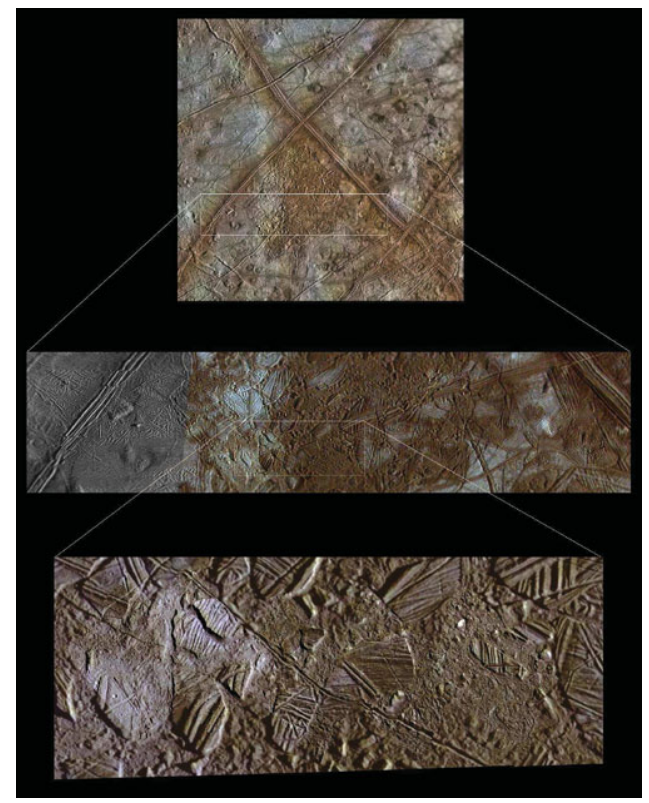

Figure 13.

\section{EUROPA: CONAMARA CHAOS}

Galileo acquired a series of progressively higher resolution mosaics of Conamara Chaos (Figure 13), the disrupted zone nestled under the crossing of the two large dark bands crossing the top scene. Conamara Chaos itself consists of numerous broken blocks of ridged plains intermingled with intensely disrupted matrix material. Erosional debris has accumulated at the base of the sheer cliffs on the edges of larger intact blocks, which stand up to 100 meters high. Small secondary craters can be resolved within the bright splotches, parts of a bright ray from the recent crater Pwyll. Chaos such as Conamara formed either by melt-through of the icy shell or more likely from diapiric blobs of warm ice that penetrated to and disrupted the surface.

TOP:

ENCOUNTER: Galileo E6 RESOLUTION: 175 meters/pixel

MIDDLE:

ENCOUNTER: Galileo E6 RESOLUTION: 55 meters/pixel

BOTTOM:

ENCOUNTER: Galileo E12 RESOLUTION: 9 meters/pixel

\section{EUROPA: RIDGED PLAINS}

The prominent double ridge, Androgeos Linea, rises 350 meters high (Figure 14). It also sits in a shallow 100-meter deep depression flanked by small cracks, formed by the weight of the ridge itself. Shear heating along faults is currently thought to be important, but the origin of the topographic ridge is unclear. Strike-slip faulting is also common on Europa, including this region, breaking the ice shell into numerous plates. Tidal deformation and internal convection move these plates around.

ENCOUNTER: Galileo E6 RESOLUTION: 20 meters/pixel 


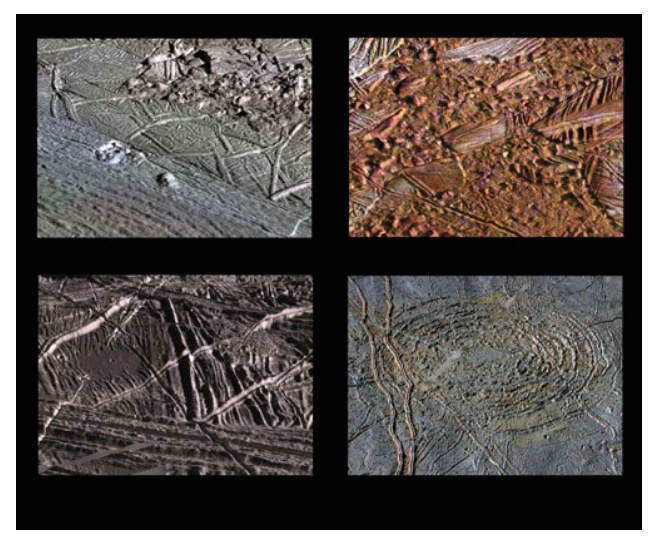

Figure 15.

\section{EUROPA: PERSPECTIVE VIEWS}

These four views show the relief of Europa in different perspectives (Figure 15). These renderings are made digitally by combining the original image mosaics with topographic maps of the surface. Vertical exaggeration in these views is 10 to 30 times normal.

\section{Upper left: Agenor Linea.}

Agenor Linea is over $1000 \mathrm{~km}$ long and has two major components: a darker southern lane and a brighter northern component. Deformation is complex, involving both compression and lateral shear, not unlike some strike-slip faults on Earth. Several small walled depressions and chaos units have subsequently altered the surface. Relief across this 40-kilometer-wide band seems to be limited to a few hundred meters.

ENCOUNTER: Galileo E17 RESOLUTION: 42 meters/pixel

\section{Upper right: Conamara Chaos.}

This perspective view shows several of the tilted fault blocks remaining within this large patch of chaos. These blocks can be up to 100 meters high. The rugged matrix material between the blocks is probably highly disrupted diapiric material and has relief of tens of meters.

ENCOUNTER: Galileo E6 RESOLUTION: 55 meters/pixel

Lower left: Ridged plains.

This mosaic show the complexity of ridged plains in some areas. Double ridges and broader ridge complexes of varying complexity are present. Some of the bands formed as new material intruded from below when the ice shell pulled apart. Several patches of ridged plains are relatively smooth and may have escaped deformation. The small smooth feature a few kilometers across appears to be liquid water that ponded here and has now frozen over.

\section{ENCOUNTER: Galileo E4 RESOLUTION: 25 meters/pixel}

Lower right: Tyre multiring impact basin This multi-ring impact structure stretches $\sim 150$ kilometers across and resembles much larger ancient impact structures on Ganymede and Callisto, such as Asgard. Tyre features concentric inner ridges and outer graben fractures, surrounded by hundreds of small secondary craters. The fractures form only if the icy lithosphere is very thin compared to the size of the impact cavity.

ENCOUNTER: Galileo E14 RESOLUTION: 170 meters/pixel 


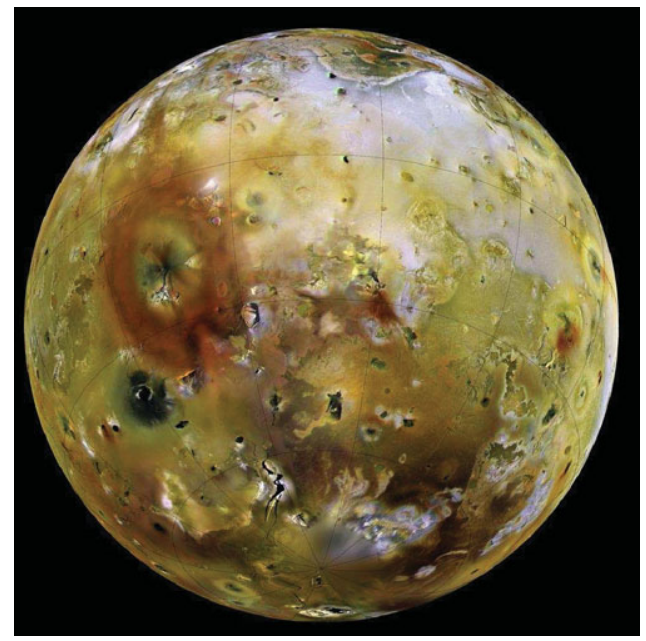

Figure 16.

\section{Io}

IO GLOBAL (SOUTHERN HEMISPHERE)

The gigantic reddish Pele and dark Babbar Patera eruption sites dominate this hemisphere (Figure 16). These huge deposits form as volcanic plumes of gas and dust fall back to the surface. Smaller reddish fan-shaped deposits betray the locations of recent or ongoing eruptions at Marduk and Culann Paterae. The different colors likely represent differences in sulfur composition. The dark deposits at Babbar probably include magnesium-rich orthopyroxenes. Reds in the Pele plume are the signature of short-chain sulfur molecules, but at polar latitudes may be due to radiation damage to short-chain sulfur molecules. Whitish materials near the South Pole are probably sulfur dioxide frosts outgassed from mountain slopes or at scarp failures. Orthographic Projection: Center $35^{\circ} \mathrm{S}, 240^{\circ} \mathrm{W}$

\section{IO - NORTH-TO-SOUTH}

The southern of these two mosaics (Figure 17) includes rugged Tohil Mons, reddish Culann Patera, the large deep caldera Michabo Patera, a bright and dark radial flow complex and small shield volcano, and a variety of pits, lineations and scarps within the intervening volcanic plains. Michabo Patera is $1.4 \mathrm{~km}$ deep and surrounded by a low mesa $\sim 100$ meters thick. Tohil Mons towers 8 kilometers high, but no relief is evident at Culann Patera. Two 2-kilometer-high shield volcanoes, including Zamama, can be seen in the northern mosaic, as well as the newly formed and very hot Thor volcanic flow complex (which was producing a 500-kilometer high plume) at the northeastern end of the mosaic.

ENCOUNTER: Galileo I32 RESOLUTION: 340 meters/pixel

\section{IO: PROMETHEUS}

First observed by Voyager in 1979 as a 100-kilometer high plume of gas and dust (Figure 18, upper left), Prometheus is one of the most consistently active volcanoes on Io, being observed in all subsequent Galileo encounters (top). The large dark lobate feature seen at higher resolution is a complex flow field composed of hundreds of overlapping flows. The bright plume itself (composed of $\mathrm{SO}_{2}$ ) forms when hot dark flows move across the surface, volatilizing the sulfur dioxide into a gas, which jets into space and back onto the surface. 


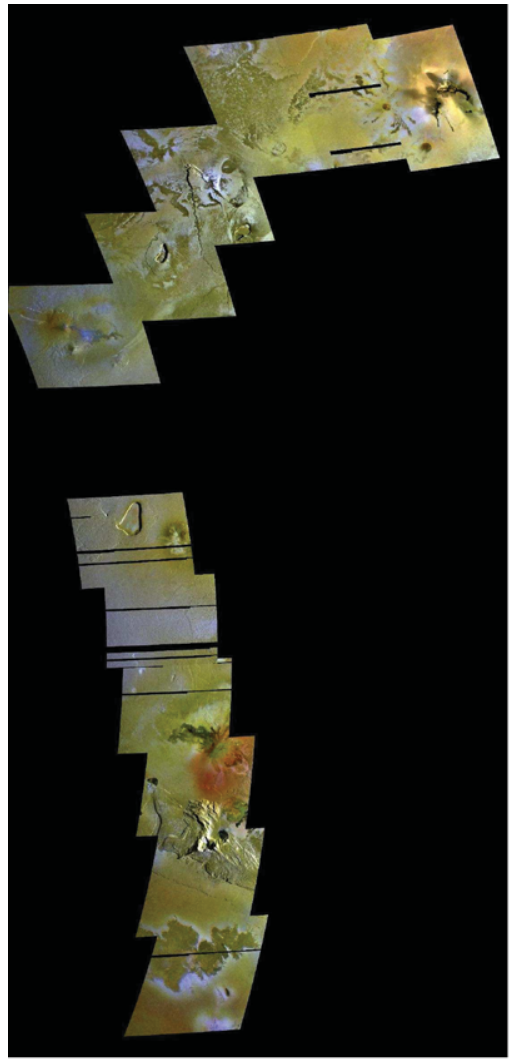

Figure 17.

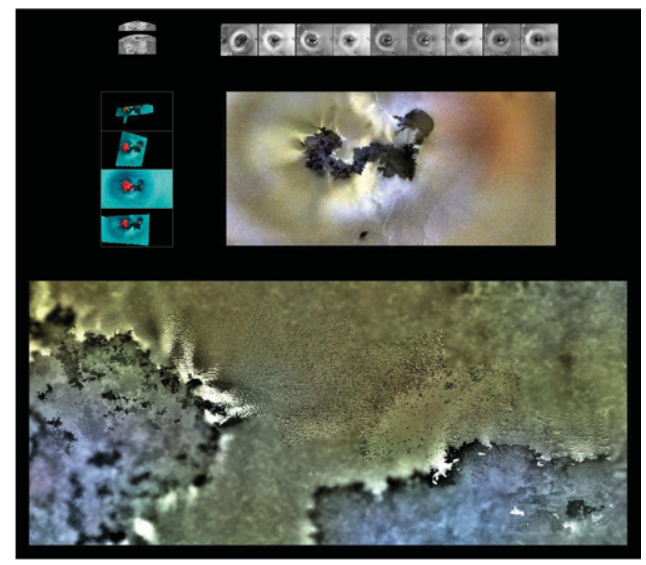

Figure 18.

ENCOUNTER: Galileo I27 RESOLUTION: 170 meters/pixel

ENCOUNTER: Galileo I27 RESOLUTION: 12 meters/pixel

IO: TOHIL MONS

Towering 9.5 kilometers above the plains, Tohil Mons is comprised of several distinct parts (Figure 19). A rectangular eastern plateau rises gently toward the west, culminating in the craggy peaks seen here. The lobate outlines of this plateau suggest it has undergone

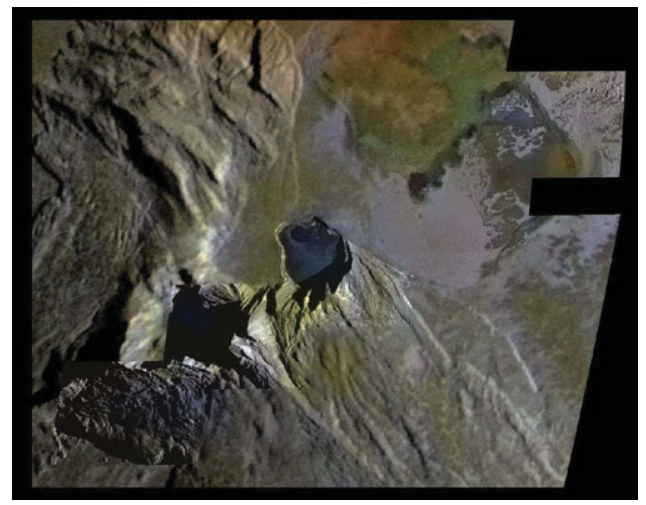

Figure 19. 


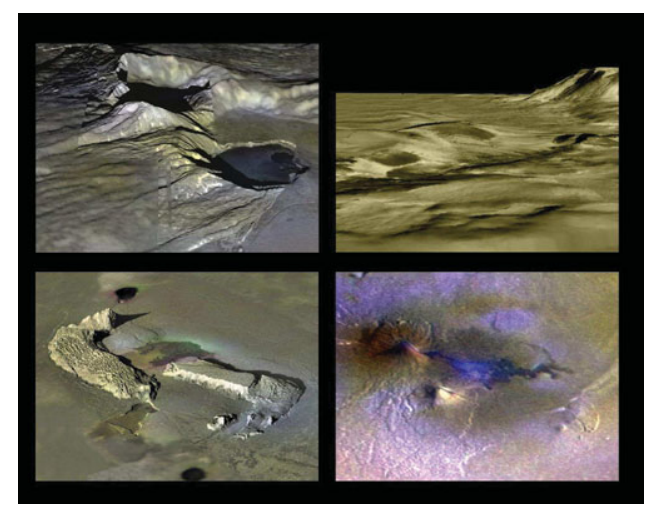

Figure 20.

partial collapse. An intensely fractured northern plateau to the north rises $\sim 3.5$ kilometers. Tohil Patera lies immediately north of Tohil Mons and includes dark and bright deposits of various colors. The smaller, dark-floored Radagast Patera, a hotspot, lies between the northern mountain and the southeastern plateau. Along the crest of Tohil Mons is a large quasi-circular amphitheatre, mostly in shadow, whose floor sits roughly 2 kilometers above the plains.

ENCOUNTER: Galileo I32 RESOLUTION: 52 meters/pixel

\section{IO: PERSPECTIVE VIEWS}

These four views show the relief of Io from different perspectives (Figure 20). These renderings are made digitally by combining the original image mosaics with topographic maps of the surface. Vertical exaggeration in these views is 10 to 30 times normal.

Upper left: Tohil Mons.

Tohil Mons is a complex mountain structure rising 8 to 9 kilometers high. This view shows the prominent axial peaks and the deep central amphitheatre. A scarp surrounds dark-floored Radagast Patera caldera in foreground 300-500 meters high. Striations on the flanks are of unknown origin.

ENCOUNTER: Galileo I32 RESOLUTION: 52 meters/pixel

Upper right: Telegonus Mensae.

This tabular mesa-like mountain stands 1.5 kilometers high. Several landslides and slumps have eroded into the edge of the cliff in the distance. Several low-lying volcanic fissures and flows formed in the foreground.

ENCOUNTER: Galileo I32 RESOLUTION: 10 meters/pixel

Lower left: Hi'iaka Montes.

Hi'iaka Montes is split into two parts. The southeastern section is a flat-topped mesa 2.5 kilometers high. Small mounds of debris can be seen along the edge, indicating the edge is slowly crumbling. The northwestern section is a complex structure featuring a ridged plateau 3 to 5 kilometers high, with a prominent set of peaks at the end rising 9 kilometers. Most mountains on Io are thrust blocks forced upward.

ENCOUNTER: Galileo I25 RESOLUTION: 270 meters/pixel 
Lower right: Zamama Of Io's 500 or more volcanoes, only these two (and one other) exhibit relief characteristic of classic shield volcanoes on Earth. These two are $\sim 2$ kilometers high and roughly 40 kilometers wide. The active dark flow extending from Zamama is likely mafic lava, and produces a plume sometimes visible from spacecraft.

ENCOUNTER: Galileo I32 RESOLUTION: 340 meters/pixel

\section{The movies}

A series of videos (http://www.youtube/com/galsat400) have also been produced from the Voyager and Galileo spacecraft data. These videos simulate what we might see if we had a mobile spacecraft capable of maneuvering at low altitudes over the surfaces of these icy and volcanic moons. The videos were produced using the image mosaics combined with topographic data produced by P. Schenk from stereo and low-Sun images, allowing us to view the relief of these moons from any perspective.

\section{The Atlas}

All images were produced by Paul Schenk, Lunar and Planetary Institute, Houston, based on Voyager and Galileo imaging data. Map images are excerpted from the Atlas of the Galilean Satellites (P. Schenk, Cambridge Univ. Press, Cambridge, 2010). The Atlas includes global maps of all four moons at 1-kilometer resolutions and all high-resolution mosaics from Voyager and Galileo spacecraft. A description of the satellites, their geologic and observational histories and the Atlas format, as well as detailed Appendices are also featured. 